\title{
Artelogie
}

Recherche sur les arts, le patrimoine et la littérature de l'Amérique latine

$9 \mid 2016$

Horizons et perspectives de la culture en Colombie (1990-2015)

\section{Figures disparues, figures de la disparition dans les œuvres de Luis Caballero et d'Oscar Muñoz}

Paul Bernard-Nouraud

\section{OpenEdition}

Journals

Édition électronique

URL : http://journals.openedition.org/artelogie/334

DOI : 10.4000/artelogie.334

ISSN : 2115-6395

Éditeur

Association ESCAL

Référence électronique

Paul Bernard-Nouraud, «Figures disparues, figures de la disparition dans les œuvres de Luis Caballero et d'Oscar Muñoz », Artelogie [En ligne], 9 | 2016, mis en ligne le 20 juin 2016, consulté le 17 novembre 2020. URL : http://journals.openedition.org/artelogie/334 ; DOI : https://doi.org/10.4000/artelogie.334

Ce document a été généré automatiquement le 17 novembre 2020.

Association ESCAL 


\title{
Figures disparues, figures de la disparition dans les œuvres de Luis Caballero et d'Oscar Muñoz
}

\author{
Paul Bernard-Nouraud
}

L'auteur remercie vivement María Margarita Malagón-Kurka et Beatriz Caballero, sceur de l'artiste en charge de son cuvre, pour leur aimable concours à la réalisation de cet article ainsi que pour avoir permis la communication de textes autrement inaccessibles.

\section{I}

1 Si l'on admet que la bidimensionnalité caractérise la peinture, laquelle occupe une superficie généralement plane à laquelle l'artiste ajoute ce qu'on pourrait désigner comme une tierce dimension, conférant ainsi à l'œuvre une contenance propre, plus ou moins évidente, plus ou moins latente, mais une contenance tout de même, alors on pourrait envisager deux directions parmi les multiples voies au travers desquelles les œuvres excèdent leurs limites superficielles : soit en surface, soit en profondeur. Dans le premier cas, en effet, dès lors que les formes mises en œuvre débordent l'espace imparti, qu'une ligne interrompue par le cadre se prolonge au-delà et avec elle l'image qu'elle délimite, celle-ci, en s'écoulant hors du terrain de l'art, paraît empiéter sur le réel et gagner alors en "épaisseur "; dans le langage typographique anglais, on dirait que l'image « saigne » de la page. Dans le second cas (et bien que, dans les faits, les deux débords puissent se combiner), le motif délinéé semble revenir au support d'où on ne le discerne qu'à demi, comme s'il tendait à s'abstraire, à se confondre avec lui, ou, pour mieux dire et quitte à risquer un néologisme, à s'y " profondre ». L'une direction étend l'image, l'autre l'intensifie.

2 Chacune à leur manière, l'œuvre de Luis Caballero et celle d'Oscar Muñoz usent respectivement de ces procédés d'extension et d'intension, et portent ainsi les images qu'ils produisent aux limites de leur disparition. Le premier n'a peint que des fragments de corps, la plupart sans visages; le second nombre de visages sans corps, 
presque tous menacés d'effacement par le support qui les absorbe ou en lequel ils se dissolvent. En première approximation, rien ne permet donc de comparer, et moins encore d'associer, les deux œuvres : la carrière de Caballero a commencé au milieu des années 1960 et l'attention presque exclusive qu'il a porté au corps humain, en particulier au nu masculin, comme le rendu traditionnel de ses figures, indiquent qu'il n'a eu de cesse d'interroger les maîtres de la Renaissance et du maniérisme jusqu'à sa mort en 1995. Muñoz a quant à lui réalisé ses premières peintures à la fin des années 1970 sous l'influence du Pop art et de l'hyperréalisme, lesquels lui ont permis d'intégrer de manière critique la photographie dont s'était toujours défié pour sa part Caballero.

En seconde approximation, cependant, on aperçoit un goût commun aux deux artistes pour un certain graphisme qu'atteste l'usage récurrent, pour ne pas dire par moments exclusif, du noir et blanc, comme si le dessin demeurait sous-jacent aux deux œuvres, et cela même lorsque Muñoz paraît s'en éloigner tout à fait avec ses "protographies " des années 2010 ou que Caballero considère ses toiles de la seconde moitié des années 1980 comme plus " picturales » et par conséquent moins « dessinées », qu'auparavant. C'est qu'en réalité le style de Caballero connaît à cette période une inflexion apparemment minime, mais qui prend un tout autre relief lorsqu'elle est mise en regard des œuvres de ses contemporains: les formes qu'il emploie, plutôt que de s'étendre, s'intensifient. En s'inspirant ici en partie de l'analyse de l'art contemporain colombien proposée par María Margarita Malagón-Kurka dans Arte como presencia indéxica (Malagon-Kurka, 2010), essai consacré notamment à l'œuvre d'Oscar Muñoz, on fait ici l'hypothèse que ce changement mineur dans l'œuvre de Caballero place celle-ci dans une situation transitionnelle entre les formes développées par l'art colombien dans les années 1950-1960 et celles qui apparaissent au cours des années 1980 et se développent au cours de la décennie suivante jusqu'à aujourd'hui.

Pour le dire de manière encore schématique, il s'opère alors un passage de représentations où les corps apparaissent souvent déformés, voire violentés, à des corps figurés disparaissant, comme si la figuration elle-même inquiétait la représentation et mettait celle-ci à l'épreuve de l'expérience. Par conséquent, la pointe de l'hypothèse que l'on formule est que l'on peut déceler dans cette instabilité des corps figurés les indices de formes nouvelles de violence qui s'abattent alors sur la Colombie, une évocation des disparus, mais aussi une mise en doute - voire en suspens - des possibilités de représenter cette mémoire conflictuelle, quitte, donc, à ce que le référent échappe à l'œuvre qui en serait informée. C'est pourquoi ce vaste mouvement de suspicion/suspension à l'égard des images par ceux-là mêmes qui les font pourrait bien finir par les emporter aussi, la révocation des images issues de l'histoire de l'art faisant pièce à leur évocation lorsqu'il s'est agi de rendre compte de la mémoire des disparus de Colombie.

\section{II}

5 Afin de saisir les implications de ces transformations diverses, il convient donc d'appréhender quelques-uns des contours de l'œuvre de Caballero. À première vue, elle est classique, voire néoclassique, non exempte aussi de quelques travers académiques. C'est que l'artiste peint effectivement des académies, des études de nus masculins extrêmement détaillées, minutieuses mêmes, où l'anatomie de chacun des muscles est d'autant plus saillante qu'elle est mise par l'artiste sous tension. Caballero peint des 
corps en extase, dont la puissance et la monumentalité proviennent explicitement des ignudi qu'a peints Michel-Ange au plafond de la Chapelle Sixtine et de L'Esclave mourant, sculpture conservée au Louvre (on retrouve dans nombre de ses nus le bandeau qui ceint la poitrine de l'esclave), et la sensualité des maniéristes, au premier rang desquels Pontormo. En recourant à ces figures, le peintre poursuit ici le périlleux rêve romantique qui consistait à modeler des figures, selon ses propres termes, "plus vives [vivas] que l'homme [...] plus pleines de sa présence ; qui arrivent à être de véritables surhommes [superseres]. Des images avec leur pouvoir propre ${ }^{1}$.» (Cité in Traba, $1984: 161)$

6 Mais un voile, pour partie issu lui aussi du romantisme, tombe déjà sur ces figures, c'est celui de Géricault, des cadavres du Radeau de la Méduse (1818-1819) ou de ses études de membres découpés copiés à la morgue de Paris; celui également de Goya dont les nus suppliciés et dévêtus que l'on voit dans les Désastres de la guerre gravés dans les années 1810 sont les seuls corps figurés à recouvrer une forme de grandeur plastique et de précision anatomique ${ }^{2}$. Si Caballero fut d'abord, comme beaucoup d'artistes latinoaméricains qui la découvrirent en 1959 à la Biennale de São Paulo, un fervent admirateur de la peinture de Francis Bacon, ce dont témoignent ses premières compositions, comme le polyptique de 1968 intitulé La Chambre de l'amour, il ne semble avoir conservé de lui que son refus de l'anecdote illustrée et retenu seulement plus tard l'idée que le hasard intervient positivement dans l'élaboration de l'œuvre.

7 Lorsqu'il reconnaît en effet dans un entretien accordé en 1992 "que le hasard est très important dans la peinture ", qu'« il faut savoir tirer parti du hasard » afin de la "conduire au hasard lui-même» (Caballero, 1992: 16), comme Bacon entendait manipuler le hasard (Sylvester, 1996:60), Caballero est déjà entré dans une nouvelle période de son art. La fragmentation des corps figurés sous forme de gros plans empêchait que leur représentation ne verse dans la narration de même qu'elle participait de leur équivoque en ce qu'on ne pouvait distinguer si leur torsion relevait de l'étreinte sexuelle ou du martyre. Mais en faisant droit au hasard, les lignes de structure de ses corps ont révélé qu'elles étaient aussi - et peut-être d'abord-des lignes de faille. Les corps figurés à partir des années 1980 ne sont pas moins monumentaux qu'avant, ils sont parfois même plus massifs encore, mais au sens géologique du terme: ses figures se minéralisent, on les arpente, elles ne sont plus cernées mais sillonnées, et pleines d'accidents qui font presque des taches (certains nus sont même parsemés de taches). Ces morceaux de corps nouvelle manière répondent aux morceaux de paysages qui, comme l'a remarqué Marta Traba, ont remplacé les plages de couleurs des décennies précédentes (Traba, 1984:p. 162) ${ }^{3}$. Ce ne sont plus seulement des corps à la renverse comme on les trouvait chez Goya ou Géricault, ils sont désormais abîmés, et ce que le cadrage serré comme l'absence des visages qui lui est corrélative ne permettait plus de distinguer, l'estompe des contours le rend cette fois quasiment indiscernable. Autrement dit, l'extension que donnait jusqu'à cette période l'artiste à ses images, allant jusqu'à des formats panoramiques, est dans les années 1980 traversée par une forme inédite d'intension.

8 Soit dit en passant, cette oblitération des corps figurés, leur résorption partielle dans les supports comme l'absence de titre de la plupart de ses œuvres à partir des années 1970 , et par conséquent d'« histoire», explique en partie que les corps figurés de Caballero soient aujourd'hui encore quelquefois jugés scandaleux en Colombie ${ }^{4}$. Sans doute, quoique de manière très ambiguë, ces images interfèrent-elles aussi sur le mode 
du sacrilège avec le fond catholique du pays. Plus profondément, cependant, la gêne qu'elles suscitent vient de ce qu'elles rappellent la mémoire récente du pays, coïncidant avec son histoire et, conséquemment, avec celle de son art. Ce rapport n'est certes pas causal, et on ne saurait trop se garder, lorsqu'il s'agit d'interpréter un exemplaire de l'art contemporain colombien, de recourir aux deux cadres interprétatifs surdéterminés par le fait qu'il s'agisse précisément d'un art fait en Colombie: l'exotisme d'une part, et la violence de l'autre, avec leur corollaire inévitable: la sensualité. Toutes choses à l'égard desquelles l'œuvre de Caballero se situe là encore à la limite, car ces facilités d'interprétation concernent potentiellement aussi bien la réception que les œuvres qui en sont l'objet. L'historien et critique d'art William Alfonso López Rosas a pu dénoncer en ce sens la «vulgate violentologique [vulgata violentológica] ${ }^{5}$ " (López Rosas, 2006) qui s'empare de tous les niveaux de l'art contemporain colombien, vulgate qui tendrait finalement, selon lui, à faire des œuvres d'art montrant la violence des objets de luxe, et dont l'exotisme aurait pour dernière conséquence la désappropriation des formes de la culture populaire nationale. Si l'on veut effectivement mesurer la véritable prégnance de la violence politique dans de telles œuvres, c'est précisément en portant attention à leurs formes, car c'est en elle que se logent les effets de l'histoire, plus durablement qu'en leurs thèmes. Le rapport entre l'œuvre et son référent est en effet d'autant plus profond qu'il est implicite, souvent latent, nébuleux presque, et qu'elle incline, en toute fin de compte, à se soustraire à son emprise.

III

Dans son essai déjà cité, Malagón-Kurka a tenté de penser l'évolution des formes mises en œuvre dans l'art contemporain colombien des années 1980 sous ce rapport à la violence. Sans entrer dans le détail de sa démonstration, on se contentera de rappeler ici les grandes lignes des transformations à la fois politiques et artistiques qu'elle repère et telles qu'on peut les déceler dans l'œuvre de Caballero d'abord, laquelle n'est pas l'objet de l'analyse de Malagón-Kurka même si elle a par ailleurs été le commissaire de plusieurs expositions monographiques à son sujet ${ }^{6}$. Sa démonstration se fonde sur le célèbre texte de Rosalind Krauss paru en 1977, "Notes sur l'index ", dans lequel l'auteure fait de l'index la structure des œuvres états-uniennes de l'époque informées qu'elles sont désormais, même lorsqu'elles sont abstraites, par le paradigme photographique (Krauss, 1985 : 79 - 90). Malagón-Kurka, quant à elle, observe en effet une tendance similaire dans l'art colombien des années 1980, en particulier dans les œuvres de Beatriz González, très proche de Caballero ${ }^{7}$, de Doria Salcedo et d'Oscar Muñoz.

L'art de la période dite de la Violence qui a ravagé le pays de 1946 à la fin des années 1950, et dont l'assassinat du dirigeant libéral Jorge Eliécer Gaitán le 9 avril 1948 marque le véritable déchaînement, se caractérisait par des œuvres très référées politiquement, et souvent influencées par les formes du Guernica (1937) de Picasso. Le Massacre - 10 avril (1948) d'Alejandro Obregón, lui-même présent au début du Bogotazo, en est l'exemple le plus connu et le tableau a rapidement acquis à l'intérieur du pays une notoriété comparable. Quelques années plus tard, on décèle cependant déjà dans Violence, qu'Obregón peint en 1962 et qui reçoit alors le premier Prix du Salon national des Artistes, une tendance intensive à l'absorption du corps figuré dans le paysage et de 
celui-ci dans le support. «Le tableau est absolument gris, écrit alors Traba, absolument sourd, absolument silencieux : pour la première fois, la tragédie a un interprète à son immense mesure.» (Cité in Malagón-Kurka, 2010:22) Sous ce rapport, les artistes postérieurs sont bien plus sûrement les héritiers de cette œuvre que de celle de 1948 . La propension à l'allusion de Violence, de même que sa palette assourdie (quoique marquée d'une tache rouge sang), n'est d'ailleurs sans doute étrangère ni à Bacon ni aux photographies qui commencent à circuler sur la Violence ${ }^{8}$. On ne sait si Obregón lui-même a emprunté son motif à l'une d'elles; il est en revanche très probable que Caballero, qui avait alors dix-neuf ans, ait vu ces clichés et à peu près certain qu'il a ensuite étudié de près Violence où les reliefs du cadavre de femme dessinent une ligne d'horizon aussi bien que de séparation entre les deux plages chromatiques, et où le corps apparaît d'autant plus mutilé qu'il est cadré très serré. À condition de ne pas perdre de vue que l'interposition des prototypes classiques maintient les figures de Caballero sous tension plus qu'elle ne les distord, et que son faire traditionnel contrebalance toujours leur aspect fragmentaire (le gros plan participant de leur monumentalité sur le mode de l'extension, on l'a dit), on peut envisager l'œuvre de Caballero comme héritière de ces figures, mais l'héritière infidèle. D'abord proche de la « néo-figuration » qui caractérise selon Traba l'art de la « post-violence » (posviolencia), son œuvre s'apparente, à partir des années 1980, à cet art indiciel (indéxico) qui définit aux yeux de Malagón-Kurka l'art contemporain colombien ; l'index pouvant être tenu, sous le rapport qu'on a décrit au début, comme un signe intensionnel, ainsi qu'on l'examinera plus en détail d'après l'œuvre de Muñoz cette fois. Peu à peu, en effet, sous le coup des événements politiques comme de l'histoire de l'art, le torso se mue en spectre chez Caballero, et devient une figure de l'après-coup.

11 Tout l'intérêt de l'essai de Malagón-Kurka tient précisément au fait qu'elle parvient à mettre en relation changements artistiques et bouleversements politiques sans penser ces relations en termes de causalité. Cela ne l'empêche pas de considérer la prise du Palais de Justice de Bogota en 1985 comme un point de rupture (même si l'événement paraît avoir davantage affecté l'œuvre de Gonzalez et Salcedo que celle de Muñoz), ni d'envisager d'après les analyses sociopolitiques de Daniel Pécaut les formes nouvelles qu'ont prises les violences en Colombie à cette époque. Si la Violence bipartite des années 1940-1950 avait été marquée par des velléités exterminatrices et, subséquemment, par la commission d'atrocités et de massacres de masse (le conflit aurait fait entre 200 et 300000 victimes), celle des années 1980 n'est certes pas moins extrême et n'a sans doute en rien soldé les comptes avec ce passé et avec " l'expérience » alors acquise, mais elle est devenue protéiforme et multidirectionnelle, et par conséquent moins représentable ou alors à découvrir de nouvelles formes.

Pécaut y a vu une banalisation de la violence, qu'elle soit d'origine politique ou criminelle, touchant tous les espaces (ruraux comme citadins) quoiqu'avec une intensité variable, au point qu'il a pu parler d'une déterritorialisation des phénomènes violents (Malagón-Kurka, 2010:36 - 37). De manière diffuse, cette propagation de la violence extrême à toutes les sphères de la société colombienne a provoqué une crise de ses représentations. Non plus, cependant, au sens où les images manquaient, comme ce fut pour partie le cas à l'époque de la Violence, la photographie que prit Sady González du cadavre de Gaitán en avril 1948 voilant alors pour partie toute autre mort en devenant le point de fixation iconique de la Violence, mais tout au contraire du fait de la profusion d'images de morts violentes; profusion qui, comme le rappelle 
Malagón-Kurka, distingue le cas colombien de ceux des dictatures chilienne ou argentines contemporaines (Malagón-Kurka, 2010 : 39).

En ce phénomène réside le paradoxe colombien relatif à la représentabilité de la violence. En rendant invisibles les images des disparus, la censure des dictatures latinoaméricaines avait certes redoublé la violence de la disparition, mais elle avait aussi maintenu, même si c'était évidemment de manière frauduleuse, à la fois une norme du visible (ce qui ne se peut regarder, pour le dire en termes goyesques, demeurant effectivement soustrait à la vue) et un principe de droit, c'est-à-dire une autre forme de normalité (en l'absence de preuve - ni corps ni image du corps disparu - le crime est réputé n'avoir pas eu lieu). Paradoxalement donc, en Colombie, la surabondance d'images montrant des corps assassinés a produit par d'autres moyens des effets semblables à ceux recherchés par les dictatures dont les œuvres d'art permettent, à rebours de cette tendance, de mesurer les conséquences à long terme. Le commissaire d'exposition José Roca a ainsi parlé d'une «escalade [escalamiento] de la tolérance visuelle » analogue, selon lui, «à la dépendance que génère une drogue fortement addictive - ou à un effet d'immunisation ». Car en effet la répétition de ces images, écrit encore Roca, «transforme la violence en quelque chose de mythique et par conséquent d'inévitable » qui confère à la violence " un caractère d'étrangeté [otredad] ${ }^{9}$ " (Roca, 2003).

14 Ainsi définie, la violence apparait, sinon naturelle, au moins culturelle, inscrite dans les ressorts profonds de la société colombienne, et non plus comme un pan aberrant de la réalité quotidienne. L'expression par laquelle le langage désigne en Colombie le sort de ceux qui ont péri de mort violente illustre cette apparente banalisation: d'eux, on dit que la terre les a avalés - "se los tragó la tierra ». La voie passive de la formule entérine d'une certaine manière la fatalité de ce qui leur est arrivé, l'acceptation de leur destin, de même que la dépersonnalisation (Pécaut parle plus largement d'une désubjectivation de la violence) reporte sur la nature - la terre - la cause de leur disparition. Cependant, il est bien évident qu'une telle expression revêt aussi une valeur conjuratoire, et que l'euphémisme fait autant saillir la monstruosité de ce dont il rend compte qu'il en atténue la portée.

Cette contradiction, inhérente aux processus de traduction des phénomènes de violence extrême excédant les possibilités même du langage, participe ainsi d'un régime de confusion entre les disparus réels, si l'on ose dire, et les morts que l'on désigne de la sorte par euphémisme, précisément; confusion qui se retrouve jusque dans les modèles qu'emploie Muñoz dans son œuvre, on va le voir. On ne saurait cependant réduire celle-ci, non plus que celle de Caballero, à une simple correspondance entre les formes qu'elles évoquent et celles prises par la violence généralisée des années 1980. Ce qui est en jeu ici, et qui fait écho de manière à la fois plus indirecte et pourtant plus significative à la réalité colombienne, c'est la mise en doute de la capacité de l'image à se fixer comme telle. La poussée intensionnelle que l'on peut observer tout particulièrement dans l'œuvre de Muñoz porte celle-ci aux limites de la disparition. En ce sens, son art paraît se tourner d'abord vers ses propres possibilités qu'il met radicalement à l'épreuve jusqu'à mettre en doute la capacité même du support artistique à soutenir durablement une image donnée, mais c'est ainsi qu'il rend compte de la prégnance du contexte dont apparemment il se détourne. 
Les différents propos tenus par Muñoz en entretien tout au long des années 2000 manifestent la tension qui traverse son œuvre, prise entre le contexte qui est le sien (l'artiste n'a jamais cessé de vivre et de travailler à Cali) et la nécessité de s'y soustraire, soustraction qu'il faudrait dans ces conditions regarder moins comme un repli de l'art sur et pour l'art que comme un jeu, une façon de retrouver le jeu propre de l'art et d'y faire jouer ce contexte, précisément, au sens où l'on dit qu'il y a du jeu entre deux éléments d'une même structure - un interstice et du mouvement.

"Il n'est pas possible d'oublier la violence qui nous entoure ", reconnaissait par exemple Muñoz en 2003 avant d'ajouter qu'il faut malgré tout « la porter sur un autre plan et l'élaborer. » (Cité in Malagón-Kurka, 2010, 99). L'année suivante, à Hans-Michael Herzog, il soutenait qu'" on ne peut parler d'une époque de la violence » en Colombie, dans la mesure où celle-ci dure toujours » (Herzog, $2004: 240$ ), allant jusqu'à avancer, lors d'une conférence donnée cette fois en 2006, que ses «travaux d'aujourd'hui partent de l'intérêt pour comprendre le mécanisme développé par une société qui a fini par vivre la routinisation [rutinización] d'une guerre, d'une succession de guerres qui ont plus de cinquante ans et qui ne se terminent toujours pas, avec de puissants secteurs de la société intéressés dans le fait de l'entretenir.» (Muñoz, 2006: 203) L'œuvre de Muñoz est pourtant loin de correspondre tout uniment à ses propres analyses et si elle devait obéir à un programme artistique, celui-ci serait plus sûrement orienté par une déclaration du type de celle qui suit, faite dès 1991, que par ses considérations proprement politiques : « la profondeur, il faut la cacher dans la surface [superficie], soutenait ainsi l'artiste. Les choses sont toujours derrière les apparences. L'empreinte [huella] cache le visible. » (Cité en note in Malagón-Kurka, 2010 : 137). Là est aussi tout le sens de la recherche intensionnelle et indicielle qui motive le travail de Muñoz, lequel alterne ainsi, depuis les années 1970-1980 jusqu'à aujourd'hui, entre avancées et retraits de et vers la réalité contemporaine.

Les premières images que réalise l'artiste au milieu des années 1970 sont déjà symptomatiques de l'attitude ambivalente qu'il nourrit à l'égard de la capacité de l'art à transposer la réalité sociale du pays. Inquilinatos, que l'on pourrait traduire par « taudis ", rend compte en 1975 en un style hyperréaliste (bien que déjà en noir et blanc du fait de l'emploi exclusif du graphite) de l'urbanisation galopante de Cali là où la série des Intérieurs (Interiores) de l'année suivante montre des lieux vides, parfois seulement occupés par une femme dont le modèle est la mère de l'artiste. D'une série l'autre, seul le thème donne aux images une valeur tantôt sociale, tantôt personnelle. On ne saurait du reste déceler entre elles de véritables différences de forme : une même atmosphère traverse des espaces à la fois intimes, voire teintés d'érotisme dans la première, et déshabités; le vide y est encore représenté, il n'a pas pénétré la matière même de l'œuvre et entamé en proportion sa capacité à faire œuvre, précisément.

19 Ce n'est en effet que dix ans plus tard, avec la nouvelle série intitulée Rideaux de douche (Cortinas de baño, 1985-1986), que Muñoz entreprend d'intensifier son œuvre en ce sens, et change pour cela radicalement de technique. La mise au point de celle-ci a d'ailleurs requis un certain temps et exigé de très nombreux essais, alors même que le résultat paraît hautement aléatoire. Il s'agissait en effet de sérigraphier au pochoir des figures sur des rideaux de plastique encore humides : l'acrylique glissait alors nécessairement du support, n'y laissant que des traces instables et presque informes, lesquelles, 
cependant, une fois les œuvres installées dans l'espace, évoquent aux yeux du spectateur la silhouette reconnaissable d'un homme ou d'une femme au bain, feignant que l'image-rideau ne dissimule le corps véritable.

À tous points de vue, Cortinas de baño apparaît aujourd'hui dans l'œuvre de Muñoz comme une création séminale. Le mode de représentation tend à devenir forme de présence, et le procédé qui fait advenir ces présences prépare ses impressions sur l'eau des années 1990 de même que les dessins préparatoires qu'il réalise alors rompent avec l'esthétique hyperréaliste de ses débuts en introduisant dans ses formes l'informe: la trace, qui en devient le motif principal. Par lui, en recourant aux traces plutôt qu'aux lignes du dessin, son œuvre se fait véritablement intensive et elle peut alors déployer sa dimension évocatoire sans risquer de verser dans l'illustration, quitte, même, à s'approcher tout près de l'abstraction. Ainsi les baigneurs de Muñoz peuvent être vus comme des figures de disparus parce que l'image qui les fait apparaitre les maintient dans l'indistinct et menace de les dissoudre tout à fait. Et cela bien qu'elles puissent tout aussi légitimement être reconnues comme les exemplaires d'une variation contemporaine sur le thème de la vénus anadyomène.

21 En recourant à la trace, qui constitue une catégorie originale d'indice en ce qu'elle n'est ni une empreinte ni une impression, ce qui la distingue aussi de la photographie (distinction lourde de conséquence dans l'œuvre de Muñoz, comme on va le voir), celleci se prémunit contre toute interprétation univoque. Dans ces conditions, il est tout à fait inattendu que ce soit dans la série la plus directement ancrée dans le contexte de la violence colombienne que l'artiste ait poussé le plus loin les potentialités inexpressives de la trace. "Tiznados fut motivée, rappelle en effet Malagón-Kurka, par les atrocités commises par un groupe de paramilitaires près de Cali » (Malagón-Kurka, $2010: 115$ ), lesquels s'étaient précisément surnommés «los Tiznados", approximativement «les noircis ", ceux qui s'enduisent le visage d'un noir de suie (tizne) afin de terroriser leurs victimes et de ne pas être reconnus d'éventuels témoins. Muñoz n'a cependant pas figuré ces assassins, mais les traces de leurs crimes et celles des cadavres que leurs actions de «nettoyage social (limpieza social) » ont laissées derrière elles.

Il a recouru pour cela, non plus au graphite, ni même au fusain, mais à la poussière de charbon, faisant usage aussi bien de ses propriétés plastiques, en l'occurrence salissante, que de sa valeur symbolique dans la mesure où le charbon, rappelle Muñoz, " fut du bois, quelque chose de vivant qui a été réduit à mort. » (Cité in Malagón-Kurka, 2010 : 115) Là se dessine de manière intensive et indiciaire, donc, puisque ayant lieu en les replis mêmes de l'œuvre, un certain ordre des choses, en l'occurrence des matières employées, rendues de cette façon solidaires de son sujet. On n'est guère éloigné non plus, sous ce rapport, de l'idée d'un engloutissement par la terre de ces figures, l'«idée de quelque chose qui sort de la terre et retourne à la terre [...], dit ainsi Muñoz, sort du fond et revient au fond. » (Herzog, 2004 : 246-247). Étalée sur du papier préalablement emplâtré que l'artiste a ensuite essuyé avec un morceau de tissu, la poudre de charbon tache la surface, révèle ses accidents et sa profondeur - ses interstices et son jeu -, en même temps que son effacement ménage un espace d'un blanc brouillon qui constitue la seule trace véritable des corps ainsi figurés au plus près du méconnaissable. Au regard de ceux que représentent à la même époque Caballero, les corps vestigiaux de Muñoz paraissent avoir franchi un degré supérieur d'intensification; ils se situent tout près de l'indiscernable cette fois, vidés qu'ils sont de toute expressivité; ce sont des Caballero dépouillés de toute geste artistique. 
Pourtant, le dépouillement qui caractérise Tiznados procède sensiblement du même constat que les nus fragmentaires que Caballero esquisse parfois sur ses toiles au fusain, c'est-à-dire là aussi au charbon de bois, constat d'un trop-plein d'images de la violence et de l'inadéquation formelle des photographies qui en rendent compte vis-à-vis de leur mise en mémoire. La position de Caballero sur le sujet est catégorique: «l'excès d'images que la photographie a déchaîné a produit une banalisation complète de l'image ", jusqu'à produire à ses yeux une "banalisation de la beauté, de l'image, de tout. » Dans cette situation, le dessin apparaît alors comme l'anti-photographie, et il est significatif que l'artiste use, pour les distinguer, de la théorie des sacrifices chère au xixe siècle. "Dessiner, déclare-t-il, c'est analyser. C'est choisir [escoger].». Or, selon Caballero, qui fait donc fonds ici de tout un pan de la critique classique, la photographie ignore ce choix, elle est intrinsèquement incapable de sacrifier le réel pour l'art : « La photographie ne peut pas rejeter [desechar], elle ne peut pas choisir : elle voit tout.» (Caballero, 1984 : 159). Et c'est en ce sens qu'elle ne peut pas bâtir, qu'elle ne saurait posséder ce "pouvoir", ainsi que l'a senti Malagón-Kurka, de l'image non photographique. "L'œuvre de Caballero, écrit-elle, contient une dimension constructive » (Malagón, 2003: 3), laquelle repose en fait sur le procédé de sélection lui-même, en tant qu'il est constitutif de ce procès de construction qui est aussi en partie fonction chez lui, et de plus en plus dans les années 1980, des matériaux qu'il choisit, et non seulement des formes qui sélectionne.

L'insatisfaction, voire la suspicion, de Muñoz à l'égard des photographies de la violence est moins radicale dans la mesure où elle ne vise pas le médium en tant que tel. Compte tenu de l'importance du photoréalisme à ses débuts comme des images photographiques dans toute son œuvre, on peut même considérer que la photographie est chez lui le socle de son art là où elle en est chez Caballero le repoussoir. En d'autres termes, les photographies jouent chez Muñoz le rôle de seuil entre la vision de la violence réelle, dont il a été à peu près épargné personnellement, et sa traduction plastique; l'artiste demeurant à la fois tributaire et critique des images photographiques ou bien télévisuelles.

De même que les milliers d'instantanés d'anonymes réalisés à Cali par des photographes ambulants (les fotocineros) que Muñoz acquit au poids après que cette pratique soit tombée en désuétude ont donné lieu en 2004 à l'«action" artistique intitulé El Puente (la plupart de ces photographies avaient été prises sur le pont Ortiz et c'est depuis le même pont qu'il les avait projetées sur la rivière), de même les images photographiques de toutes natures, aussi bien journalistiques, nécrologiques que réalisées par des amis photographes, qui conforment à la fois son imagier personnel et un imaginaire collectif ont-elles assuré à l'ensemble de son œuvre une assise documentaire. En un sens barthésien, Muñoz voit en effet dans la photographie le moyen de donner à ses dessins "une charge de documentalité [una carga de documentalidad]", la considérant comme un médium au sens premier du terme. "Je n'avais pas encore vraiment d'intérêt conceptuel pour la photographie, explique-t-il ainsi à propos de son appétence précoce pour elle, je la voyais plus comme un intermédiaire entre le monde et le dessin, une traduction qui conférait un caractère nouveau de documentarisme atmosphérique à mon travail. » (Wills Londoño, $2014: 84$ ) 

où Gerhard Richter élabore son Atlas, que de déployer depuis ces images son œuvre selon " une logique anti-archivistique » (Gaztalbide, 2015 : 147), où la mémoire est à la fois ordonnée et inquiétée par la retraduction des «documents» originaux. L'inquiétude provient alors du fait que les «actions» comme les œuvres de Muñoz mettent en évidence "l'impossibilité d'un confinement de l'image [impossibility of an image containment]» (Gaztalbide, 2015 : 148), laquelle excède en effet le présent en y déversant le passé - les passés. Dans cette perspective, il importe peu de savoir si les personnes figurant sur les photographies que détourne Muñoz sont des disparus au sens que l'on a donné à ce terme en Amérique latine ou bien en son sens euphémique, qu'il s'agisse de personnes tuées pour des raisons politiques ou non, l'artiste lui-même entretenant sur le sujet une certaine confusion ${ }^{10}$. Dans tous les cas, en effet, « la terre les a engloutis ", dans la mesure où l'accumulation de leurs images a fini par enfouir jusqu'à leur souvenir. postérieures de Muñoz en général entendent rendre aux événements qui en forment donc l'amorce une visibilité à laquelle nuisent, selon l'artiste, les clichés des photojournalistes dont il s'inspire. Ces images, écrit-il, «partent des photographies de cadavres faites sous l'implacable lumière du flash et publiées dans les pages rouges de la presse ", or, "ces photographies, ajoute-t-il, qui, avec cet éclairage, prétendent mettre tout en évidence [evidenciarlo todo], aplanissent et invisibilisent à la fois [aplanan e invisibilizan a la vez]. » (Muñoz, 2006 : 202) L'enjeu qui motive leur reprise est donc de ruiner ces images afin d'en reconnaître les restes, les vestiges et les traces, lesquels peuvent éventuellement faire droit aussi à ces "petits indices et détails du sol qui entourent le cadavre dans ces images» (cité in Malagón-Kurka, 2010, 115), mais qui, plus profondément, vise à rendre en partie obsolète la photographie ; l'obsolescence de l'image chez Muñoz portant à un niveau plus profond encore la caducité des corps figurés de Caballero.

Cette tendance à considérer l'image donnée comme un fardeau et son opération de traduction artistique comme un moyen de la dépouiller de son « imagéité », si l'on peut dire, est un trait trop récurrent de l'art contemporain pour que l'on puisse ici en décrire ne serait-ce que quelques-uns des innombrables prolongements ${ }^{11}$. Ce que l'on peut retenir, en revanche, c'est que Muñoz altère l'empreinte photographique en la transformant en trace, c'est-à-dire en signe informe (potentiellement méconnaissable donc) et non reproductible (la reproduction d'une trace devenant ipso facto une empreinte). Ces traces, comme les corps tracés de Caballero, quoique dans une moindre mesure, on l'a dit, sont ainsi envisagées comme des répliques au trop-plein d'image aussi bien qu'à leur netteté qui tend finalement à opacifier ce qu'elles prétendent pourtant rendre évidentes. Ce n'est pas tant que la figure évanescente, qui « a perduré comme leitmotiv dans ses œuvres » soit concomitante d'une diminution de «l'usage de photographies comme sources visuelles» (Malagón-Kurka, 2010: 107), ainsi que le soutient Malagón-Kurka, mais davantage que ces sources se dissolvent à mesure qu'il s'est éloigné de la facture photographique de ses débuts, poussant le départ jusqu'à invoquer, comme l'a aperçu Roca, « la photographie sans l'utiliser comme support de l'image. » (Roca, 2014 : 10)

29 En l'occurrence, Roca commente des images que Muñoz a regroupées sous le terme de «protographies », insistant au passage sur l'ambivalence de leur situation à l'égard du 
dessin et de la photographie : à la fois d'avant et d'après cette dernière. Roca eût préféré le terme de " protophotographie » en ce que ces œuvres obligent à revenir à l'archè du dispositif photographique, pour le dire en les termes de Jean-Marie Schaeffer dans L'Image précaire (désignation qui conviendrait d'ailleurs assez aux images que Muñoz réalise), soit à reconsidérer finalement leur pragmatique (Schaeffer, 1987). Car en effet, «à strictement parler, écrit Roca, l'image photographique existe indépendamment de là où elle est fixée ». (Roca, $2014: 14$ ) Or c'est précisément ce qui fait sa précarité, ce qui définit sa substance processuelle, et, en définitive, sa propension à la disparition.

Parmi les protographies de Muñoz, la série des Narcisses (Narcisos), entamée en 1994 et poursuivie jusqu'au début des années 2000 , est sans doute la plus emblématique de ce questionnement radicale de la formation des images et de la menace de dissolution qui pèse sur elles. Les premières œuvres montrent seulement le résultat du procédé employé : dans un caisson empli d'eau, Muñoz a dessiné à sa surface, au pochoir et à la poudre de charbon, un visage, le sien, lequel, après évaporation de l'eau, s'est déposé sur le support disposé au fond du bac, parfois de simples pages de journaux. Pour ses derniers Narcisses, l'artiste a filmé le processus en question et le spectateur voit l'image du visage se dissoudre plutôt que de se déposer au fond d'un évier tout à coup débondé, et le visage disparaître avec elle. Dans les deux cas, on assiste en fait à une déposition de l'image ; convoquée, elle se voit révoquée par cela même qui la fait advenir ; la présence de l'image entame d'autant sa capacité à représenter le réel, et c'est pourquoi MalagónKurka insiste sur la «présence indiciaire » de ce type d'œuvres afin de la distinguer de leur fonction représentationnelle.

31 Mais la remontée archéologique qu'autorise les protographies de Muñoz, aussi bien vers la photographie que vers le dessin, et par-là jusqu'à la peinture elle-même ${ }^{12}$, comme la déposition de l'image qui en résulte et qui vaut pour une quasi-destitution, n'a été rendue possible qu'en raison du contexte de crise de la représentation de la violence qui distingue le cas colombien mais vaut bien entendu hors de ses limites géographiques et historiques. L'artiste lui-même formule cette critique qui constate, d'une part, que « jamais auparavant l'image visuelle n'avait atteint par le moyen de la technologie le degré de netteté [nitidez] et d'exactitude qu'elle a aujourd'hui», et, d'autre part, que "jamais auparavant on n'avait autant mis en doute sa capacité à montrer la réalité » (Cité in Malagón-Kurka, 2010 : 120). La mise en obsolescence, pour ne pas dire en crise, des médias de la représentation constitue, sous ce rapport, la réplique elle-même critique à cette situation. Le dispositif de mise en œuvre redevient archaïque et l'image qu'il produit, plus qu'un relevé du réel, se présente désormais comme sa déposition, aux sens à la fois documentaire (si ce n'est judiciaire) et esthétique du terme.

Cette fragilisation de l'acte artistique explique les titres de certaines des œuvres les plus récentes de Muñoz comme ses Impressions faibles (Impresiones débiles) de 2011 qui convoquent, selon ses propres mots, «trois photographies mythiques de la mémoire politique du pays » (Wills Londoño, $2014: 108$ ), parmi lesquelles le cliché du cadavre de Gaitan, tout en les altérant jusqu'à presque les effacer - user, jusqu'à les révoquer en les évoquant, donc. Muñoz réalise d'ailleurs la même année Sédimentations (Sedimentaciones) dont la forme, des clichés blancs projetées sur fond noir, rappelle explicitement celle des planches de l'Atlas Mnémosyne d'Aby Warburg à laquelle Muñoz emprunte aussi sa dynamique "polychronique » et analogique, à ceci près que les images qui sont tour à tour plongées dans le bain, au lieu d'y être "arrêtées » (on dit en espagnol « revelar » 
pour " développer ») s'y dissolvent avant de réapparaître dans un bain jumeau sur une feuille vierge. Ainsi passent et repassent infiniment ces images qui ne sédimentent que dans l'œil de celui qui le regarde, c'est-à-dire fugitivement.

Ce jeu permanent entre le visage (rostro) et la trace (rastro), l'image et sa dérobade, se fonde bien sur le contexte de violence extrême qui sous-tend l'histoire de la Colombie et son actualité. Néanmoins, il s'en dédit aussi et rejoint plus largement une mise en question des pouvoirs de l'image dont les formes sont non seulement indiciaires mais intensionnelles. L'absence y apparaît comme un mode de présence, allusif et détourné, et la trace comme signe que la disparition a eu lieu; ce lieu fût-il désormais seulement celui de l'œuvre envisagée comme un «réceptacle de preuves» [receptáculo de evidencia]» (Malagón-Kurka, 2010:34), ou, pour mieux dire, comme un lieu de recueillement.

\section{BIBLIOGRAPHIE}

ÁlVAREZ L., (2008). « Disolvencia y fantasmagoría ». Oscar Muñoz: Documentos de la amnesia, Jiménez, Carlos (dir.), Badajoz, Museo Extremeño e Iberoamericano de Arte Contemporáneo, , pp. 161-171.

(1984). « Luis Caballero », Pintado en Colombia, Séville, Junta de Andalucía.

(1992). « Entrevue en noir et blanc avec Luis Caballero ». Luis Caballero, Paris, Union latine, pp. 14-21.

CABALLERO L., (2014). ¡Pobre de mí, no soy sino un pobre pintor ! : cartas de Luis Caballero a Beatriz González, Bogota, Universidad Jorge Tadeo Lozano.

GAZTAlBIDE M. C., (2015). « An Anti-Archival Logic ». Contingent Beauty: Contemporary Art from Latin America, Ramirez, Mari Carmen (dir.), Houston, Museum of Fine Arts, pp. 147-151.

GRAHAM A. J., (2012). « Assisted Breathing: Developing Embodied Exposure in Oscar Muñoz's 'Aliento'« . New York, Latin American Perspectives, vol. 39, n 3, mai, pp. 63-73.

KENTRIDGE W., (2002). "Some Thoughts on obsolescence : Artist Questionnaire : 21 Responses". Cambridge, October, vol. 100, printemps, pp 16-18.

KRAUSS R., (1993). « Notes sur l'index ». L'Originalité de l'avant-garde et autres mythes modernistes Paris, Macula, coll. « Vues ». [1985, tr. de l'anglais par J.-P. Criqui]

LOPEZ ROSAS W. A., (2006). « Entre el compromiso artístico y el populismo estético ». Bogota, Universidad Nacional Periódico, $\mathrm{n}^{\circ}$ 97, septembre [en ligne : http://

historico.unperiodico.unal.edu.co/ediciones/94/15b.htm]

MALAGon María Margarita, (2003). Luis Caballero y el poder de la imagen, Bogota, Galerie Alonso Garcés.

MALAGON-KURKA M. M., (2010). Arte como presencia indéxica : la obra de tres artistas colombianos en tiempos de violencia : Beatriz González, Oscar Muñoz y Doris Salcedo en la década de los noventa, Bogota, Universidad de los Andes, Facultad de Artes y Humanidades. 
MUÑoz O., (2008). « Presentación en Global. Photography Now, Tate Modern ». Oscar Muñoz :

Documentos de la amnesia, Jiménez, Carlos (dir.), Badajoz, Museo Extremeño e Iberoamericano de Arte Contemporáneo, , pp. 201-204.

SCHAEFFER J.-M., (1987). L'Image précaire. Du dispositif photographique, Paris, Seuil, coll. « Poétique ».

SYLVESTER D., (2005). Entretiens avec Francis Bacon, Paris, Skira. [1996, tr. de l'anglais par M. Leiris et M. Pepiatt]

ROCA J., (2003). « Ausencia/Evidencia : José Alejandro Restrepo, Oscar Muñoz, Teresa Margolles ». Bogota, Columna de Arena, $\mathrm{n}^{\circ}$ 48, 23 janvier [en ligne : http://universes-in-universe.de/columna/ col48/col48.htm].

RODRIGUEZ AMAYA F., (2003). «Colombia : Arte y poder en el siglo xx ». Toulouse, Caravelle, juin, n $20-21$, pp. 107-127.

SELEANU A., (2007). « Art contemporain en Colombie ». Montréal, Vie des Arts, vol. 51, pp. 20-37.

TRABA M., (1984). « Otra estación en el infierno ». Pintado en Colombia, Séville, Junta de Andalucía, , pp. 161-164.

Wills LoNDoÑo M., (2014). « Entretien avec Oscar Muñoz ». Oscar Muñoz. Protographies, Paris, Jeu de Paume, Filigranes, Bogota, Museo de Arte del Banco de la República, , pp. 81-111. Pau Paumes Paume, Filigranes, Bogota, Museo de Arte del Banco de la República, , pp. 81-111.

\section{NOTES}

1. Sauf mention contraire, toutes les traductions sont de l'auteur.

2. On pense en particulier aux planches $27,33,37$ et 39 de la série, intitulées respectivement Caridad, ¿Que hay que hacer más ?, Esto es peor, et ¡Grande Hazaña! ¡Con muertos!

3. On peut rappeler le rôle central qu'a joué Marta Traba dès la fin des années 1950 (elle fonde le musée d'art moderne de Bogota en 1957) et jusqu'à sa mort brutale en 1983 dans un accident d'avion, non seulement pour la promotion de l'œuvre de Caballero, dont elle fut aussi la professeure, mais pour celle de l'art contemporain colombien en général. L'essai qu'elle a fait paraître en 1965, intitulé Les Quatre monstres cardinaux (Los cuatro monstruos cardenales), dans lequel elle analyse les œuvres de Francis Bacon, de Willem de Kooning, de Jean Dubuffet et de José Luis Cuevas, a fortement contribué à la découverte de ces artistes en Colombie et favorisé l'émulation artistique du pays. Pour une approche plus critique de l'influence de Traba sur le milieu artistique colombien, et notamment bogotain, au cours de cette période, cf. Rodríguez Amaya, 2003 : 118 sqq.

4. Cela n'implique pas, loin s'en faut, que l'œuvre de Caballero ait été marginalisée. Traba l'a exposée dès 1966 alors qu'il n'avait que 23 ans, et il est décédé à Bogota en 1995 où il était revenu de Paris où il vivait et travaillait à l'occasion d'une importante rétrospective ; une salle lui est depuis longtemps entièrement consacrée dans les collections permanentes musée de la Banque de la République de Bogota et un prix à son nom a été créé dès 1996.

5. $C f$. également sur ce sujet le dossier de Vie des Arts qui n'évite pas cependant certains des écueils que l'on décrit (Seleanu, 2007). On peut rappeler que la question des relations entre les arts plastiques et la violence a fait l'objet d'une exposition à Bogota dès 1958, organisée par la Société économique des amis du pays, puis en 1999 au musée d'art moderne.

6. Notamment au musée d'art moderne de Medellin en 2013, exposition intitulée «El tormento y el deseo en Luis Caballero ».

7. Les lettres de Caballero à González ont paru en 2014 (cf. Caballero, 2014). 
8. Un premier volume issu des travaux de la Commission d'enquête parlementaire paraît cette même année 1962 ; le volume complet du rapport est publié en 1968. Malagón-Kurka, qui accorde une grande importance aux photographies reproduites dans ses ouvrages quant à leur impact sur l'art de l'époque, est plus réservée sur la connaissance qu'avait pu en avoir Obregón lorsqu'il a réalisé Violencia. Sur ces points, cf. Malagón-Kurka, 2010 : 19-21.

9. D'après l'article de Roca, cf. également Álvarez, 2008.

10. Muñoz dit en effet s'intéresser d'abord au fait que les photographies qu'il utilise montrent des personnes mortes, "pas nécessairement pour des raisons de violence politique » (Herzog, 2004 : 246) Il a également suggéré que le rapport qu'entretient une œuvre comme Aliento (1995) à l'égard du thème des disparus, "avec la charge et le sens que nous donnons ici à ce terme ", n'était pas tout à fait intentionnel. (Wills Londoño, 2014, 103) Comme l'a noté Malagón-Kurka, c'est pourtant bien en tant que figures de disparus (desaparecidos) et non comme les simples inconnus (desconocidos) qu'ils sont qu'elles ont été généralement interprétées par la critique étrangère (cf. sur ce point Malagón-Kurka, 2010, 141-142, notes). Il semble d'ailleurs que ce malentendu, si c'en est vraiment un, puisque Muñoz a par exemple participé en 2007 à une exposition intitulée The Disappeared / Los desaparecidos au Museo del Barrio de New York, ait trouvé des prolongements jusque dans la critique universitaire, $c f$. notamment Graham, 2012: 65 et 71 .

11. On pense en particulier à des artistes contemporains sud-africains ayant eux aussi travaillé sur la mémoire de la violence héritée de l'Apartheid comme Marlene Dumas, dont la dernière grande exposition en 2014 (Fondation Beyeler de Bâle et Stedelijk Museum d'Amsterdam) s'intitulait précisément L'Image comme fardeau (The Image as Burden), ou bien comme William Kentridge, dont les dessins sont parfois très proches de ceux de Muñoz, avec lequel il partage aussi une propension aux dispositifs archaïques et à la mise en obsolescence des moyens plastiques. Cf. notamment sur ce point Kentridge, 2002.

12. On peut en effet rappeler que, dans son De Pictura, Leon Battista Alberti fait de Narcisse «l'inventeur de la peinture ».Cf. sur ce point Alberti, 1435 : Livre ii, §25, 101.

\section{RÉSUMÉS}

En portant attention à l'œuvre de Luis Caballero d'abord, à celle d'Oscar Muñoz ensuite, cet article entend mettre en évidence les processus de figuration des disparus dans l'art contemporain colombien du tournant des années 1990 jusqu'à aujourd'hui. Les corps que figure Caballero à cette période montrent en effet une tendance intensive là où ils avaient d'abord semblé s'étendre au-delà des limites du tableau; tendance intensive qui informe en revanche tout l'œuvre de Muñoz. Cette forme est voisine du paradigme indiciaire qu'a énoncé Rosalind Krauss à propos de l'art états-unien des années 1970 et que María Margareta Malagón-Kurka a transposé au contexte colombien cité, à ceci près qu'à partir des corps qu'il figure il produit des traces davantage que des indices, dans la mesure où leur figuration même est menacée de disparition.

Centrando nuestra atención primero sobre la obra de Luis Caballero, y luego sobre aquella de Oscar Muñoz, este artículo quiere poner de relieve los procesos de figuración de los desaparecidos en el arte contemporáneo colombiano en el cambio de los años 1990 hasta hoy. De hecho, los cuerpos que representa Caballero muestran una tendencia intensiva la cual había 
tendido previamente a sobrepasar los límites del cuadro. Dicha tendencia se destaca en toda la obra de Muñoz. Esta forma se acerca al paradigma indéxico que enunció Rosalind Krauss al respecto del arte estadounidense de los años 1970 y que María Margareta Malagón-Kurka transpuso al contexto colombiano, con la tenue diferencia que a partir de los cuerpos figurados se producen rastros más indiciales, en la medida de que su figuración misma se ve amenazada por la desaparición.

INDEX

Palabras claves : desaparecidos, Colombia, arte contemporáneo, Muñoz (Oscar), Caballero (Luis) Mots-clés : disparus, Colombie, art contemporain, Muñoz (Oscar), Caballero (Luis)

\section{AUTEUR}

\section{PAUL BERNARD-NOURAUD}

EHESS-CRAL 